\title{
Ensemble docking revisited
}

\author{
Oliver Korb*, S Bowden, T Olsson, D Frenkel, J Liebeschuetz, J Cole \\ From 5th German Conference on Cheminformatics: 23. CIC-Workshop \\ Goslar, Germany. 8-10 November 2009
}

In recent years, the importance of considering induced fit effects in molecular docking calculations has been widely recognised in the molecular modelling community. While small-scale protein side-chain movements are now accounted for in many state-of-the-art docking strategies, the explicit modelling of large-scale protein motions such as loop movements in kinase domains is still a challenging task. For this reason ensemble-based methods have been introduced taking into account several discrete protein conformations in the conformational sampling step. Our protein-ligand docking approach GOLD [1,2] has been extended to search such conformational ensembles time-efficiently. The performance of the approach has been assessed on several protein targets using different scoring functions. A detailed analysis of pose prediction and virtual screening results in dependence of the number of protein structures considered in the conformational ensemble will be presented and limitations of the approach will be highlighted.

Published: 4 May 2010

References

1. Jones G, Willett P, Glen RC: Molecular Recognition of Receptor Sites Using a Genetic Algorithm with a Description of Desolvation. J Mol Biol 1995, 245:43-53.

2. Jones G, Willett P, Glen RC, Leach AR, Taylor R: Development and Validation of a Genetic Algorithm for Flexible Docking. J Mol Biol 1997, 267:727-748.

doi:10.1186/1758-2946-2-S1-P25

Cite this article as: Korb et al:: Ensemble docking revisited. Journal of Cheminformatics 2010 2(Suppl 1):P25.

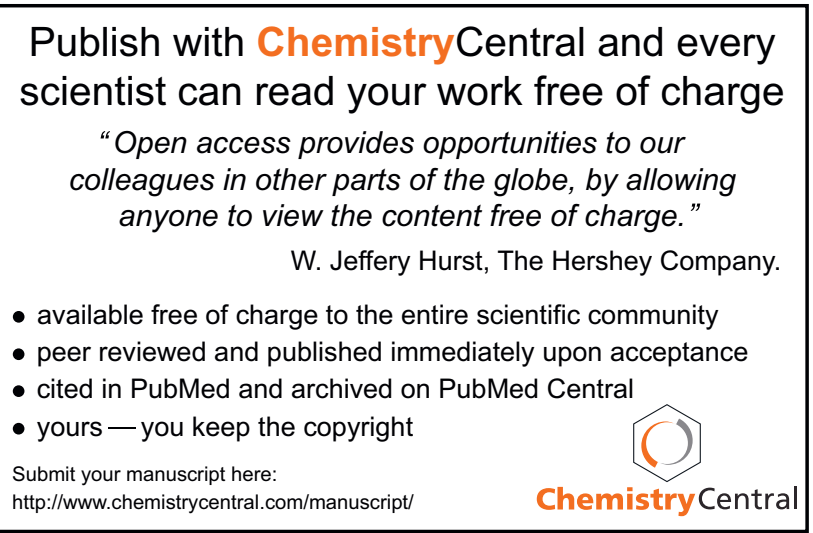

